Review Article

\title{
Current Progress and Challenges for Skeletal Muscle Differentiation from Human Pluripotent Stem Cells Using Transgene-Free Approaches
}

\author{
Nunnapas Jiwlawat, ${ }^{1}$ Eileen Lynch, ${ }^{1}$ Jeremy Jeffrey ${ }^{(D},{ }^{1}$ Jonathan M. Van Dyke, ${ }^{1}$ \\ and Masatoshi Suzuki iD ${ }^{1,2}$ \\ ${ }^{1}$ Department of Comparative Biosciences, University of Wisconsin, Madison, WI, USA \\ ${ }^{2}$ The Stem Cell and Regenerative Medicine Center, University of Wisconsin, Madison, WI, USA \\ Correspondence should be addressed to Masatoshi Suzuki; masatoshi.suzuki@wisc.edu
}

Received 21 December 2017; Revised 11 February 2018; Accepted 18 February 2018; Published 11 April 2018

Academic Editor: Zhaohui Ye

Copyright (c) 2018 Nunnapas Jiwlawat et al. This is an open access article distributed under the Creative Commons Attribution License, which permits unrestricted use, distribution, and reproduction in any medium, provided the original work is properly cited.

\begin{abstract}
Neuromuscular diseases are caused by functional defects of skeletal muscles, directly via muscle pathology or indirectly via disruption of the nervous system. Extensive studies have been performed to improve the outcomes of therapies; however, effective treatment strategies have not been fully established for any major neuromuscular disease. Human pluripotent stem cells have a great capacity to differentiate into myogenic progenitors and skeletal myocytes for use in treating and modeling neuromuscular diseases. Recent advances have allowed the creation of patient-derived stem cells, which can be used as a unique platform for comprehensive study of disease mechanisms, in vitro drug screening, and potential new cell-based therapies. In the last decade, a number of methods have been developed to derive skeletal muscle cells from human pluripotent stem cells. By controlling the process of myogenesis using transcription factors and signaling molecules, human pluripotent stem cells can be directed to differentiate into cell types observed during muscle development. In this review, we highlight signaling pathways relevant to the formation of muscle tissue during embryonic development. We then summarize current methods to differentiate human pluripotent stem cells toward the myogenic lineage, specifically focusing on transgene-free approaches. Lastly, we discuss existing challenges for deriving skeletal myocytes and myogenic progenitors from human pluripotent stem cells.
\end{abstract}

\section{Introduction}

Recent advances in stem cell biology hold great promise for use in treating and modeling neuromuscular diseases [1]. Neuromuscular diseases affecting the function or development of skeletal muscle can arise directly via muscle pathology or indirectly via disruption of the nervous system. Despite devastating consequences, no effective treatment strategies exist in many cases, including muscular dystrophy. Attractive therapeutic strategies include the replacement of affected muscle cells with healthy myocytes or progenitor cells, thereby restoring skeletal muscle function.

Human pluripotent stem cells (PSCs), which include embryonic stem cells (ESCs) and induced pluripotent stem cells (iPSCs), represent a robust cell source for developing cell-based therapies targeting degenerating muscles as well as modeling neuromuscular disease conditions and for drug screening in culture. Particularly, iPSC technology allows creation of patient-derived stem cells, which can simulate pathophysiological conditions in vitro [2]. These in vitro models are expected to work as a unique platform for drug screening and allow comprehensive study of disease mechanisms.

In the last decade, a number of culture methods for myogenic differentiation from human PSCs have been published [3]. These include (1) transgene methods employing the direct manipulation of gene expression and (2) transgene-free methods employing pharmacologic inhibitors and agonists 
as well as isolated cytokines or other protein-based signals [3]. In this review, we discuss relevant pathways and events during skeletal muscle development which have been studied and manipulated in an effort to derive myogenic cell types from human PSCs. We then overview recent progress of the methods for myogenic derivation from human PSCs, specifically focusing on transgene-free approaches. Finally, we discuss the limitations and potential of these approaches for future treatment and modeling of neuromuscular diseases.

\section{Skeletal Muscle Development and Molecular Networks}

\subsection{Embryonic Myogenesis and Terminal Differentiation into} Myofibers. During early embryogenesis, the formation of skeletal muscle begins when the paraxial mesoderm segments form somites in response to signals from the notochord, neural tube, and surface ectoderm [4]. The developing somite then forms the dermomyotome, myotome, and sclerotome. The cells in the dermomyotome express the paired box transcription factors Pax3 and Pax7 [4-7]. The dorsomedial and ventrolateral portions of the dermomyotome give rise to the epaxial (primaxial) and hypaxial (abaxial) myotomes, respectively. Myf5-positive cells in the epaxial myotomes differentiate and form the trunk and back muscles. In contrast, MyoD-positive progenitors delaminate and migrate from the hypaxial myotome into the developing limb as the source of limb muscles. Myf5 and MyoD are expressed in committed muscle cells and are located in the myotome, which is formed from the maturation of dermomyotome lips [8-10].

The terminal differentiation of progenitors and myoblasts initiates when myogenic progenitors in the dermomyotome stop dividing and exit the undifferentiated stage (Figure 1). Pax3- and/or Pax7-positive proliferating progenitors withdraw from the cell cycle once the differentiation step is initiated. These progenitors then become committed myoblasts expressing Myf5 and/or MyoD and form the nascent myotubes expressing myogenin and myosin heavy chain (MHC) (Figure 2(a)). Two waves of myotube formation occur during skeletal muscle development, sequentially giving rise to primary and secondary myotubes $[4,11]$. Primary myotubes are generated from the fusion of early myoblasts and are aligned between muscle tendons to form the basis for embryonic muscle development. Late-stage myoblasts proliferate alongside primary myotubes and fuse to form secondary myotubes. As the secondary myotubes form, motor axons begin to innervate the embryonic muscle [11]. Single-nucleated myoblasts then fuse with the nearby myotubes to form multinucleated myotubes. Thick-myosin and thin-actin filaments within the myotube begin organizing and form sarcomeres, the functional units of muscle contraction. Sequential chains of sarcomeres, called myofibrils, align in maturing myotubes. Mature myotubes contain wellorganized and aligned myofibrils which give rise to the characteristic striated pattern of skeletal myocytes (Figures 2(b) and $2(\mathrm{c}))$.
2.2. Signaling Molecules for Myogenesis. Myogenesis is delicately regulated by signaling events that influence proliferation and differentiation of stem cells and progenitor cells [4]. These events are driven by paracrine and/or autocrine signaling molecules that pattern and generate specific cellular lineages. A number of signaling molecules have been characterized to play critical roles for specification and differentiation from the somite to the myotomes $[12,13]$. Signaling molecules can also contribute to terminal differentiation of myoblasts and myotube formation. These molecules regulate the expression of myogenic genes and proteins and influence the growth and fusion of MHC-positive myotubes. This section will introduce several signaling molecules critical for myogenesis; however, this is not an exhaustive list.

Wnt signaling plays a significant role in the development of myogenic progenitors in the somite and the formation of committed myoblasts in later stages of myogenesis. A diverse family of Wnt proteins is secreted from the neural tube and ectoderm. Wnt $[12]$ and Wnt3a [14, 15] are produced in the dorsal neural tube, while Wnt7a is expressed in the dorsal ectoderm [12], and Wnt5a is localized in the dorsal ectoderm and limb mesenchyme [14]. Wnt ligands bind to Frizzled (Fzd) receptors and take action through a canonical ( $\beta$-catenin) pathway or noncanonical pathways [16]. In mouse explant cultures, Wnt1 can enhance Myf5 expression and affects epaxial muscle formation. In contrast, Wnt7a promotes MyoD expression and influences hypaxial myogenesis [12, 17]. The initial expression of Pax3 and Myf5 was decreased in mice lacking both Wnt1 and Wnt3a [15]. A Wnt antagonist Frzb1 inhibits myogenesis in presomitic mesoderm, but not in mature somites. When Frzb1 was injected in a pregnant mouse, the process of myogenesis was disturbed by the reduction of Myf5 expression [18]. An inhibitor of $\mathrm{Wnt} / \beta$-catenin signaling (IWR1-endo) inhibits myotube formation in murine myotube culture [19]. Additionally, an inhibition of glycogen synthase kinase $3 \beta$ (GSK $3 \beta$ ) can promote mesoderm differentiation via activating Wnt pathways [20-22].

Sonic hedgehog (Shh) is secreted from the notochord and floor plate of the neural tube [23] and regulates myogenic progenitor proliferation and differentiation [24]. In zebrafish, the number of Pax3- and Pax7-positive cells was significantly increased by a knockdown of the Shh gene [24]. Shh shows positive effects on muscle development by directing progenitor cells to Myf5-/MyoD-positive committed myocytes in the myotome by downregulating Pax3/Pax7 expression [25]. A reduced level of Myf5 expression was observed in Shh-null mice, resulting in a loss of distal limb structures [26]. Shh also enhances myogenic differentiation by increasing MyoD expression. An implantation experiment using Affi-Gel agarose beads soaked with $100 \mu \mathrm{g} / \mathrm{ml} \mathrm{N}$-Shh in the lumen of the neural tube showed that Shh activates both MyoD and a sclerotomal marker, Pax1, in quail embryos [27]. Shh also promotes sclerotome formation while inhibiting dermatome formation [23].

Fibroblast growth factors (FGFs), including FGF2 (or basic FGF, bFGF), are critical factors for controlling proliferation and differentiation of myogenic progenitors and myoblasts during myogenesis. FGF2 is known to inhibit 

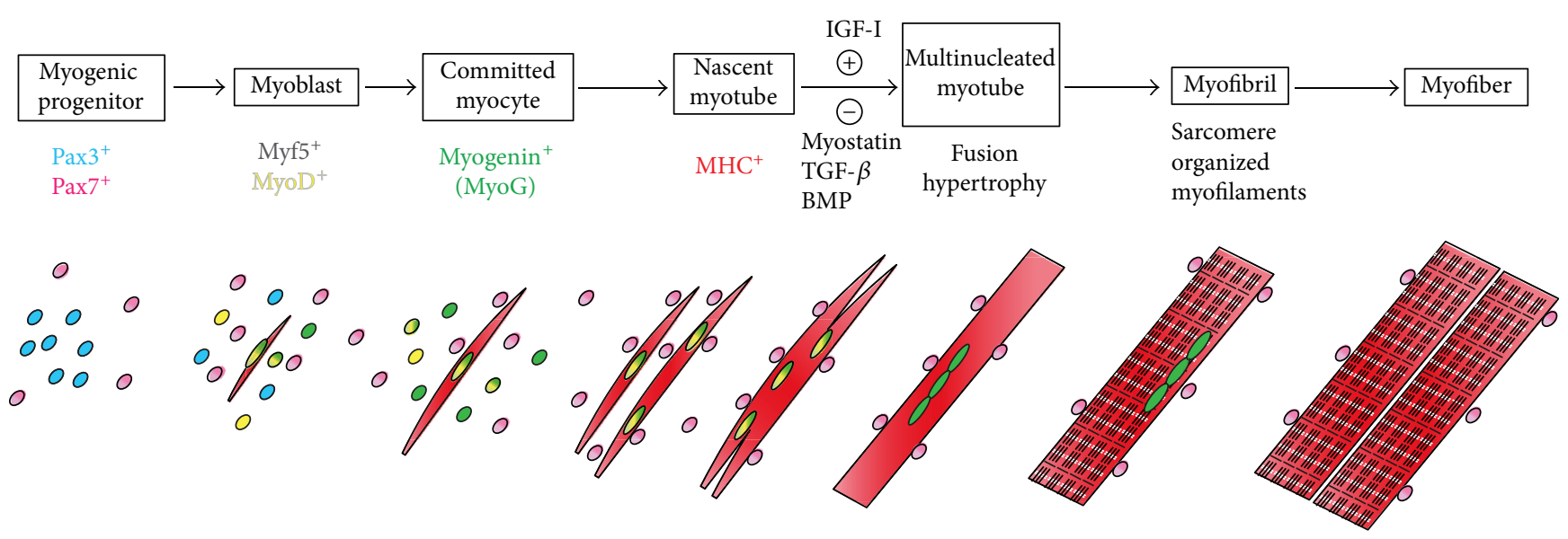

FIgURE 1: Skeletal muscle differentiation in vitro. The terminal differentiation starts when Pax $3^{+}$and/or Pax $7^{+}$progenitors begin to express Myf5 or MyoD as committed myoblasts. These myoblasts gradually express myogenin (MyoG) and form single-nucleated nascent myotubes with myosin heavy chain $\left(\mathrm{MHC}^{+}\right)$. Insulin-like growth factor-I (IGF-I), TGF- $\beta 1$ inhibitor, and myostatin inhibitors induce myotube fusion to form multinucleated myotubes. Actin, myosin, and elastic myofilaments are arranged to form organized sarcomeres within the myotubes. Organized sarcomere structures give rise to a striated pattern in the myotubes and represent the functional contraction unit of muscles.

the differentiation of myogenic progenitors into myotubes $[28,29]$, implying that FGF2 could be used to maintain the progenitors at an immature stage. Interestingly, in murine myoblast $\mathrm{C} 2 \mathrm{C} 12$ cells, inhibition of the mitogen-activated protein kinase (MAPK) pathway, which is downstream of FGF, increased the expression of MyoD, myogenin, and MHC and led to more myoblast fusion [29]. Both paracrine and autocrine effects of FGFs are proposed, as myocytes have been found to express both FGF ligands and FGF receptors. FGF ligands can bind to four FGF receptors (FGFR1-4) with varying levels of affinity. FGFR1-4 are transmembrane tyrosine kinase receptors capable of activating various downstream signaling cascades. FGFR1, 2, and 4 are expressed in immortalized myoblast cell lines such as mouse Sol 8 cells. Inhibitory effects of myocyte differentiation by FGF molecules were only observed when FGFR1 and 2 were presented in Sol 8 cells. Myogenic differentiation was stimulated when FGFR1 signals were inhibited by overexpressing truncated FGFR1 molecules [28]. Another study using chromatin immunoprecipitation-on-chip analyses demonstrated that FGFR4 is a direct downstream target of Pax3 in mouse embryo [30]. Further studies are necessary to elucidate which FGFRs are involved in modulating myogenesis. In addition, application of FGF2 or forskolin to C2C12 mouse myoblasts resulted in phosphorylation and activation of cyclic AMP response binding (CREB) protein. A gain-of-function mutation in CREB increased myoblast proliferation [31], indicating involvement of CREB signaling in myogenesis. Loss of CREB activity significantly decreased Pax3, Myf5, and MyoD expression in mouse embryos [17].

Both bone morphogenetic protein 4 (BMP4) and Notch enhance progenitor proliferation but inhibit muscle differentiation [25]. BMP4, secreted from the lateral plate mesoderm, sustains Pax3 expression and delays Myf5 and MyoD expression in chicken embryos [32]. An increased level of a BMP4 inhibitor Noggin in the dorsomedial lip of the dermomyotome of chick embryos inhibits BMP signaling and increases medial, rather than lateral, somite patterning [33]. Noggin-soaked bead implants promote muscle differentiation in chick embryos [34]. While BMP4 works as a secreted factor, an activation of Notch signaling requires direct cell-cell contacts. The Notch receptor is a singlepass transmembrane protein. Notch ligands bind to the extracellular domain of the receptor and then lead to proteolytic cleavage at the intracellular domain. After the intracellular domain is released, it migrates toward nucleases and modulates the expression of downstream genes [35]. A subset of migrating neural crest cells expresses a Notch ligand, Delta1. When chick embryo dermomyotomal cells transiently contact Delta1-expressing cells, expression of Myf5 and MyoD is activated. However, a prolonged contact with Delta1-expressing cells reverses the myogenic process resulting in Pax7-positive progenitor cells [36]. Notch signaling increases proliferation of myogenic progenitors but inhibits muscle differentiation by blocking MyoD transcriptional activity [37].

Transforming growth factor beta (TGF- $\beta$ ) and a TGF- $\beta$ superfamily protein, myostatin, are known to modulate myogenic differentiation. TGF- $\beta$ inhibits myogenic differentiation by suppressing the activity of myogenin [38]. However, a potent and selective inhibitor for TGF- $\beta$ type I receptor (SB431542) and retinoic acid have been shown to rescue the negative effect of TGF- $\beta$ on $\mathrm{MHC}^{+}$myotube formation in $\mathrm{C} 2 \mathrm{C} 12$ mouse myoblasts [39]. In mouse embryonic stem cells, a combination of TGF- $\beta$ inhibitor (SB431542), a Wnt activator (BIO), and a Shh inhibitor (erismodegib) increased the expression of Pax7, Myf5, MyoD, and myogenin and the number of $\mathrm{MHC}^{+}$myotubes [40]. Myostatin (also known as growth and differentiation factor-8, GDF-8) affects muscle cell differentiation in a manner similar to that of TGF- $\beta$. Dorsomorphin and LDN193189, which inhibit myostatin activity, significantly enhance myotube formation when 
Pax3 MyoG MH(

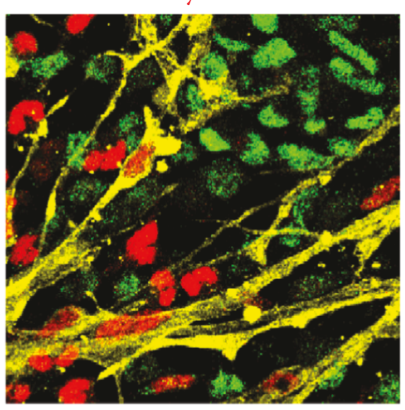

(A)
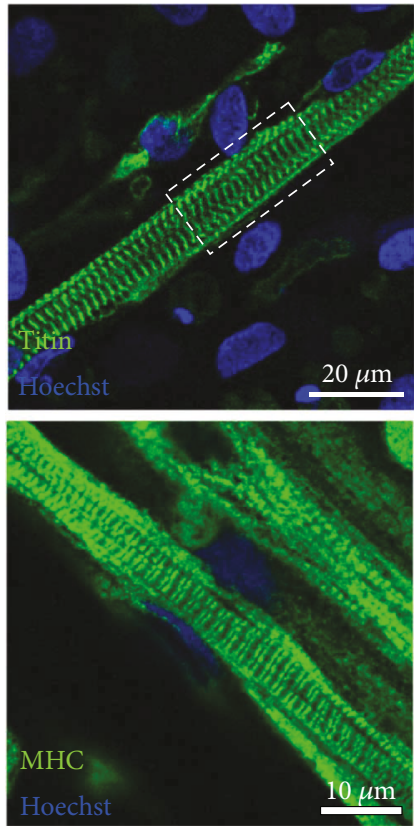

(b)
Pax7 MyoG MHC

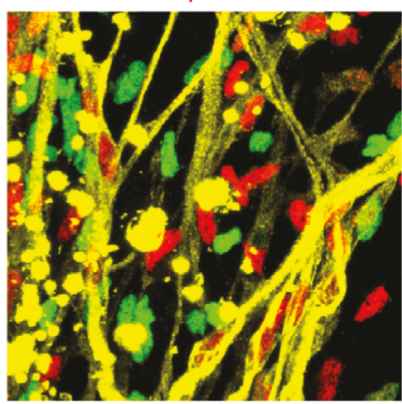

(B)
MyoD MyoG MHC

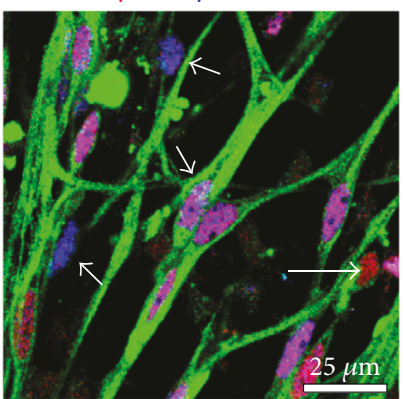

(C)

(a)
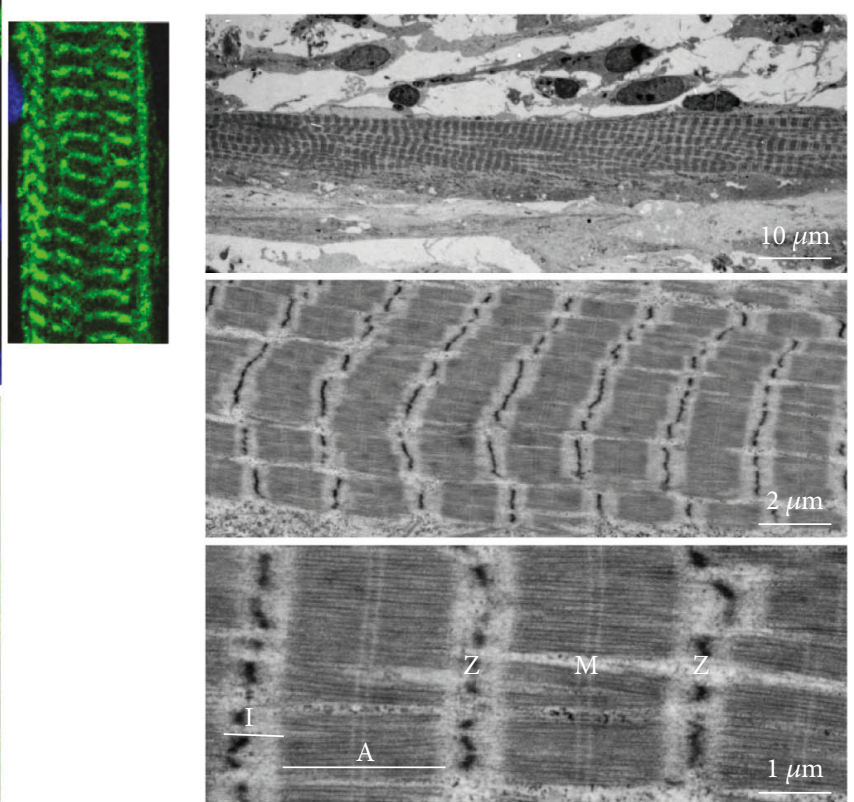

(c)

FIGURE 2: Derivation of skeletal myocytes and matured myotubes from human iPSCs using a transgene-free protocol. Human iPSCs can be sufficiently differentiated into myogenic progenitors and myotubes in a defined culture without genetic modification using free-floating spheres (EZ spheres) [59, 67]. (a) Human iPSC-derived myotubes were labeled with multiple myogenic proteins Pax3, Pax7, MyoD, myogenin (MyoG), and myosin heavy chain (MHC), demonstrating colocalization of those proteins in the same field. Some MyoD ${ }^{+}$nuclei were overlapped with $\mathrm{MyoG}^{+}$nuclei and fused on $\mathrm{MHC}^{+}$myotubes (double arrow: $\mathrm{MyoD}^{+} / \mathrm{MyoG}^{+}$). The other nuclei were not overlapping with MHC but expressed either MyoD or MyoG (arrow: MyoD ${ }^{+} / \mathrm{MyoG}^{-}$; or arrow head: $\left.\mathrm{MyoD}^{-} / \mathrm{MyoG}^{+}\right)(\mathrm{C})$. Neither Pax3 ${ }^{+}$ nuclei (A) nor Pax7 $7^{+}$nuclei (B) showed any localization with $\mathrm{MyoG}^{+}$nuclei, which mostly fused on $\mathrm{MHC}^{+}$myotubes. (b) Sarcomere formation in iPSC-derived myotubes. Titin staining revealed that striated patterns were clearly visible in the myotubes at 12 weeks MHC staining in the same cell preparations used for titin labeling. (c) Ultrastructures of iPSC-derived myotubes. After 12 weeks of terminal differentiation, mature sarcomeres were observed to be assembled into myofibrils. Morphological hallmarks, including I-band of actin filaments and A-band with distinct $\mathrm{M}$ line across myosin filaments, were clearly visible. Sarcomere $\mathrm{Z}$ lines appeared to be reasonably aligned and gave rise to a striated pattern. This figure is reproduced from Jiwlawat et al. [67] (under the Creative Commons Attribution license/public domain).

applied to primary human myotubes and murine myotubes [41]. Follistatin, another myostatin inhibitor, increased fusion index and myogenic protein expression (including MyoD, Myf5, and myogenin) in C2C12 cells [42]. Another myostatin inhibitor, growth and differentiation factorassociated serum factor protein 1 (GASP-1), also enhances myogenin expression and fusion index in myotubes differentiated from C2C12 cells [43].
Insulin-like growth factor-I (IGF-I) is produced and secreted from myogenic cells and regulates muscle differentiation and growth. Both IGF-I receptors and IGF binding proteins are dramatically increased in mouse C2 myoblast cells during muscle differentiation [44]. IGF-I triggers terminal differentiation of myoblasts through the MAPK signaling pathway and increases protein expression of myogenin in murine C2C12 myotubes [29]. IGF-I, but not IGF-II, 
promotes myofiber fusion and hypertrophy in avian myotubes. This hypertrophy was promoted by increased synthesis and lower degradation of MHC proteins [45]. Interestingly, the steroid testosterone can stimulate fusion and hypertrophy of primary human myotubes via the IGF-I signaling pathway [46].

\section{Derivation of Skeletal Muscle Cells from Human PSCs}

Cell signaling plays a critical role in all stages of myogenesis. The timing of expression and the levels of signaling molecules are tightly controlled in order for the different stages of myogenesis to occur smoothly [12, 13]. Accumulated knowledge of the signaling pathways guiding myogenesis has aided the creation of a number of methods for deriving myogenic progenitors and myocytes from human PSCs. Current methods can be broadly categorized into two approaches: (1) induction of myogenic differentiation by overexpression of myogenic genes (transgene methods) and (2) derivation of myogenic progenitors under defined culture using growth factors and/or signaling molecules without transgenes (transgene-free methods).

3.1. Transgene-Based Approaches to Enhance Myogenic Differentiation. Selective induction of myogenic genes, such as the overexpression of PAX3, PAX7, and MYOD1, has been used in order to increase the efficiency of myogenic differentiation [3]. As discussed above, these transcription factors play critical roles in promoting proliferation and differentiation of myogenic progenitors and myoblasts during embryonic myogenesis. Different systems of gene expression, such as lentiviral and piggyback-based approaches, have been applied to transduce PAX7 [47, 48] and MYOD1 [49-52] genes into human PSCs. The transcription of myogenic genes can also be controlled by inducible gene expression systems such as tetracycline or tamoxifen [47-52]. These progenitors can be sufficiently enriched by fluorescence-activated cell sorting (FACS) if the transgene construct contains a fluorophore reporter gene like green fluorescent protein (GFP) and mCherry $[47,49]$.

One notable advantage of the transgene method is that transgene-based approaches can secure high efficiency of progenitor preparation (more than $90 \%$ in several methods). Typically, transgene methods yield progenitors more rapidly than transgene-free methods. However, as these approaches require an introduction of exogenous genes to the cells, the resulting cells may not fully reflect the normal processes of progenitor proliferation, differentiation, and maturation. Additionally, genetic modification remains a regulatory concern if the progenitors are to be used for cell-based therapy in patients. As such, myogenic progenitors prepared by transgene-free methods may be more suitable for transplantation in patients.

3.2. Transgene-Free Approaches: Myogenic Derivation under Defined Culture Conditions. Recent attempts have been made to derive myogenic progenitors from human iPSCs and ESCs under defined culture conditions using specific molecules secreted as paracrine factors that play important roles in muscle development (Table 1). These molecules control proliferation, migration, and differentiation from mesodermal cells into somite and dermomyotome [25]. FGF2 has been used at varying concentrations $(5-100 \mathrm{ng} / \mathrm{ml})$ to direct and enhance myogenic differentiation [20, 53-61]. Although 10$20 \mathrm{ng} / \mathrm{ml}$ FGF2 is commonly used to maintain proliferation in cell lines or primary cells, during our recent study, we found that a high concentration of FGF2 (100 ng/ml) significantly increased the number of Pax7-positive myogenic progenitors from human PSCs [59]. Other growth factors such as insulin-like growth factor-I (IGF-I), epidermal growth factor (EGF), hepatocyte growth factor (HGF), and platelet-derived growth factor (PDGF) have also been known to promote myogenic progenitor expansion and differentiation in human PSCs [57]. IGF-I can enhance myotube hyperplasia and fusion $[62,63]$. IGF-I has been used at a concentration of $2-50 \mathrm{ng} / \mathrm{ml}$ to enhance terminal differentiation $[55-57,61,64]$.

Small molecule inhibitors have also been used to direct and enhance myogenic differentiation. GSK3 $\beta$ inhibitors, such as CHIR99021 [55, 61] and BIO (6-bromoindirubin$3^{\prime}$-oxime) [20], can promote mesoderm induction during differentiation by activating Wnt pathways. CHIR99021 significantly enhances the expression of mesoderm genes such as T, TBX6, and MSGN1 in human PSCs $[54,55,65]$, indicating that this selective GSK3 $\beta$ inhibitor can promote mesoderm differentiation. While CHIR99021 has proven useful for in vitro mesoderm differentiation, it should be noted that it should only be used in culture for short periods and at a low concentration due to its toxicity $[54,55]$. In fact, a longer exposure (more than $3 \mathrm{mM}$ for 4 days) or a higher concentration (10 $\mu \mathrm{M}$ for 2 days) of CHIR99021 results in toxicity in human PSC cultures $[54,55]$. By contrast, a potent and reversible GSK $3 \beta$ inhibitor (BIO) demonstrates the lowest toxicity among other GSK3 $\beta$ inhibitors [20]. Further, an adenylyl cyclase activator, forskolin, has been used in a triple cocktail with FGF2 and a GSK3 $\beta$ inhibitor (BIO) to promote muscle differentiation [20].

Inhibitors of BMP type I receptors or TGF- $\beta$ type I receptors, such as LDN193189 [56, 61, 64] and SB431542 [57], have been used to enhance derivation of a myogenic population from human PSCs. In some protocols, basal medium supplement of insulin-transferrin-selenium (commonly known as ITS) has been used to induce the initial step of mesodermal specification $[53,55,66]$. Oncostatin, necrosulfonamide, ascorbic acid, insulin, and dexamethasone were recently used in combination with growth factors and TGF- $\beta 1$ inhibitors to increase skeletal myocyte derivation efficiency. These small molecules promoted a high percentage of skeletal muscle differentiation (up to $70 \% \mathrm{MHC}^{+}$ myotubes) and shortened the differentiation period to less than a month [57]. A Notch antagonist DAPT ( $\gamma$-secretase inhibitor) increased MyoD and myogenin gene expression [65]. A combination of CHIR99021 and DAPT synergistically enhanced myogenic differentiation [65]. Additionally, the rescue effect of LDN193189 and SB431542 mixture was demonstrated by the reduction of BMP4 levels and an increase of fusion index when applied to myotubes prepared from patient iPSCs with Duchenne muscular dystrophy [65]. 


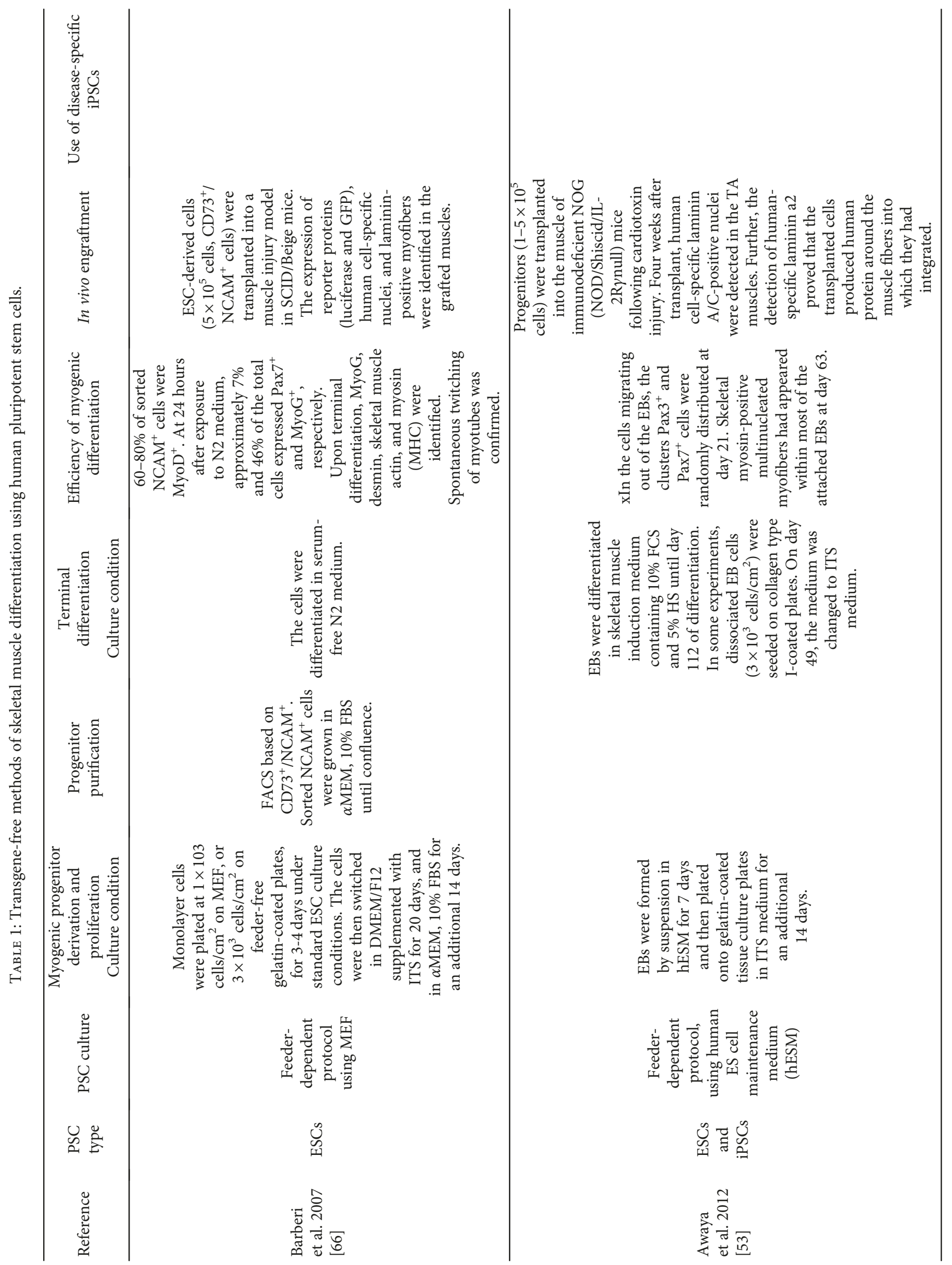




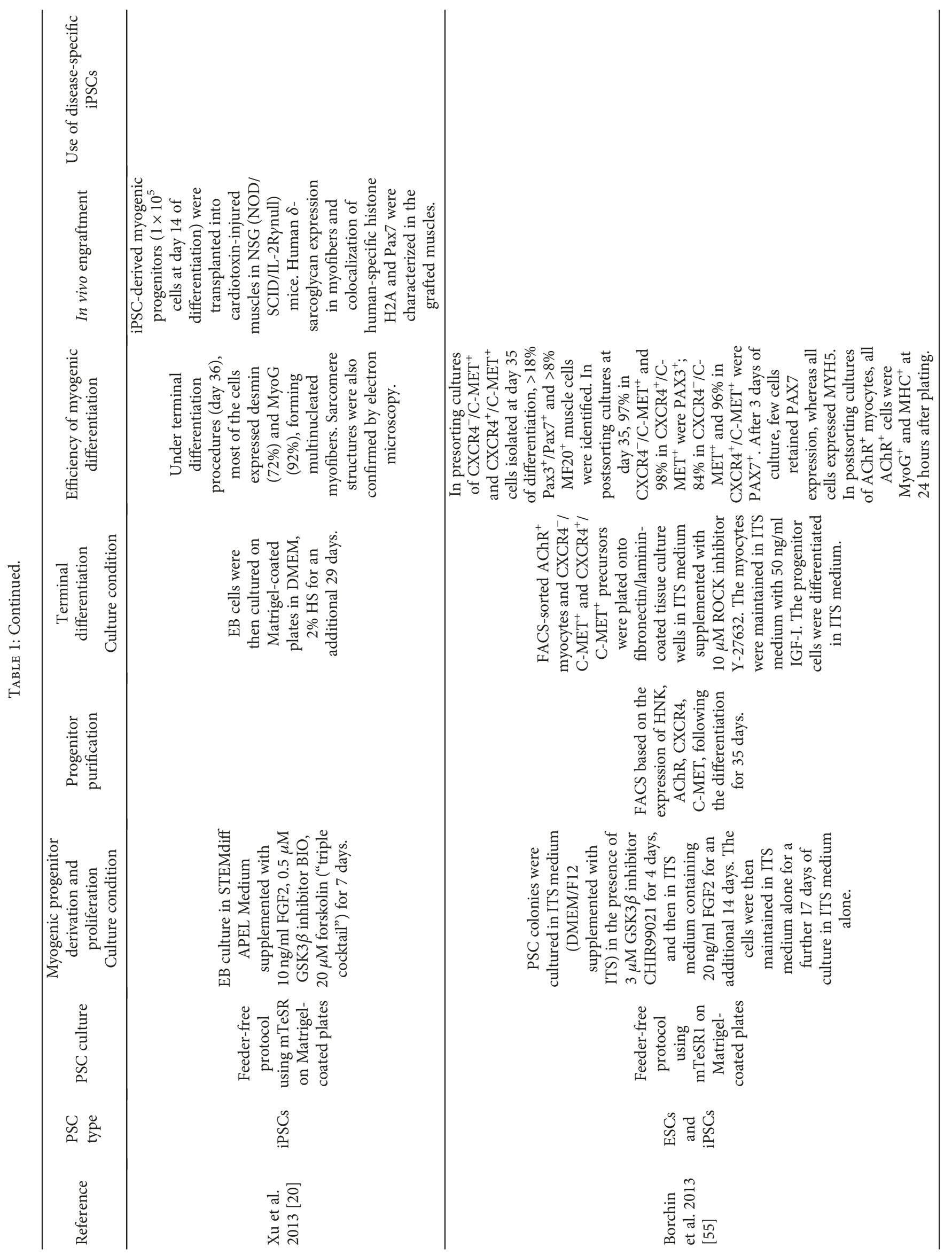




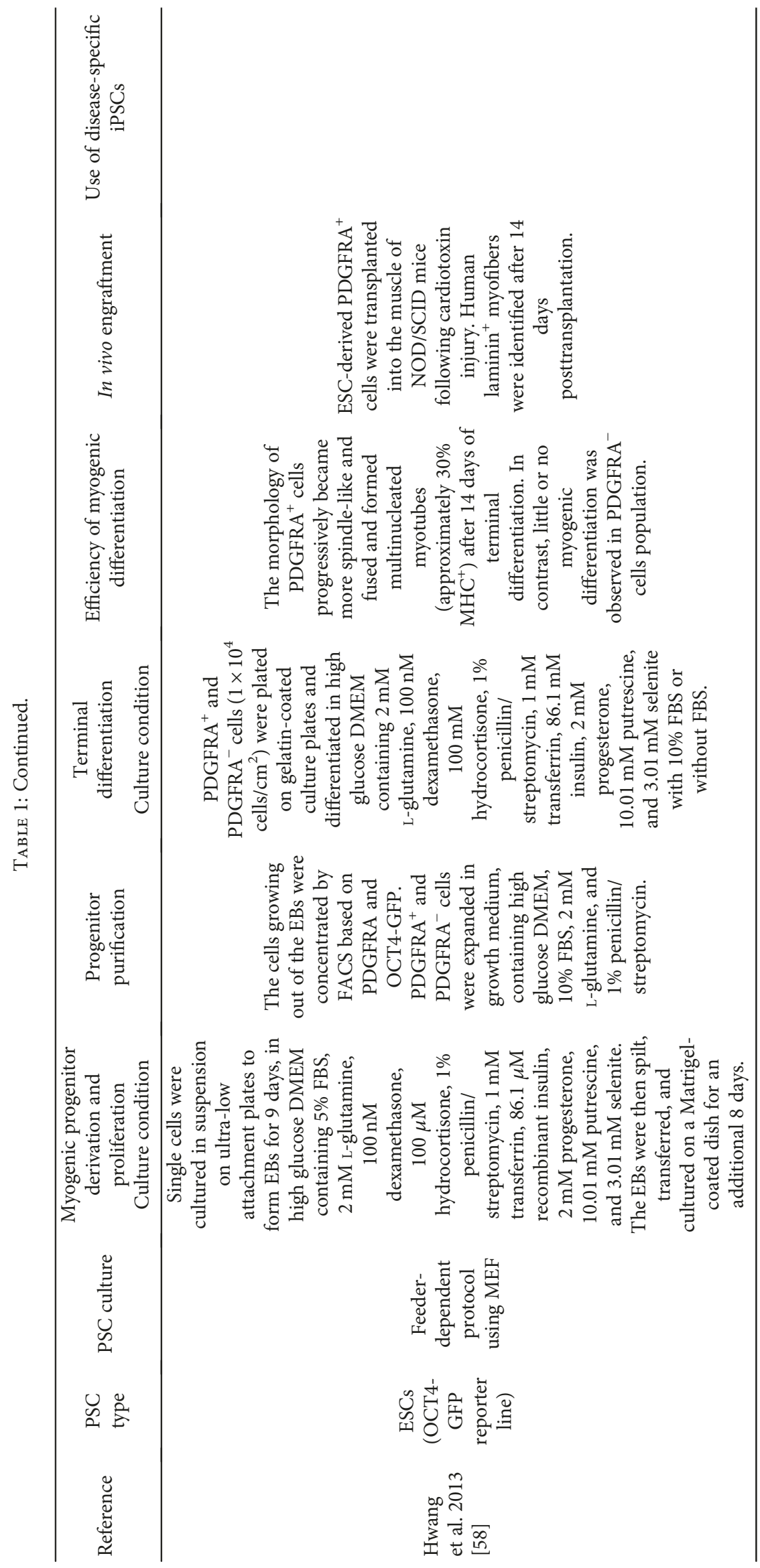

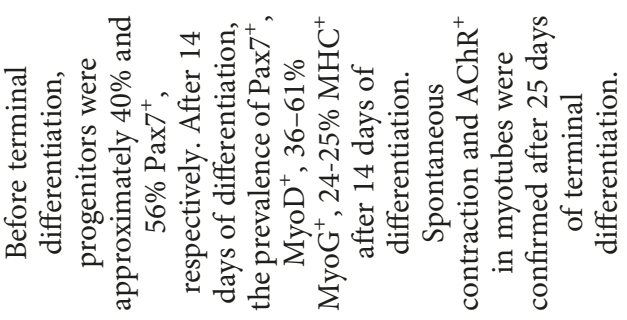

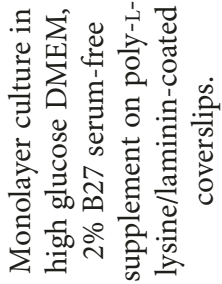

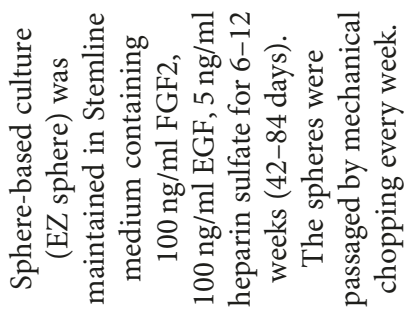

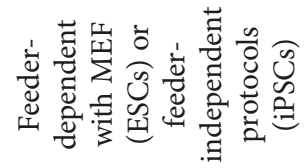

चี

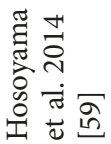




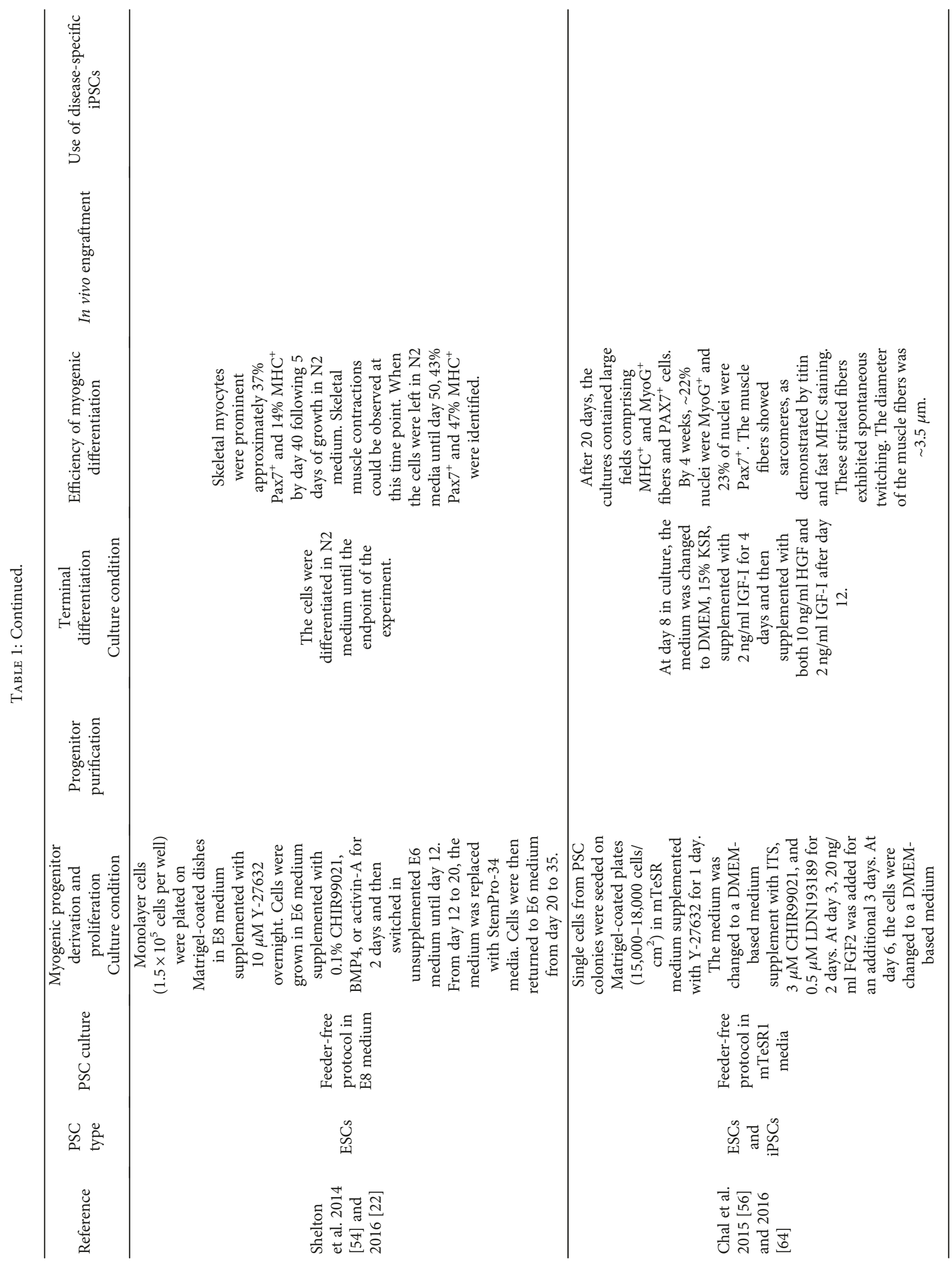




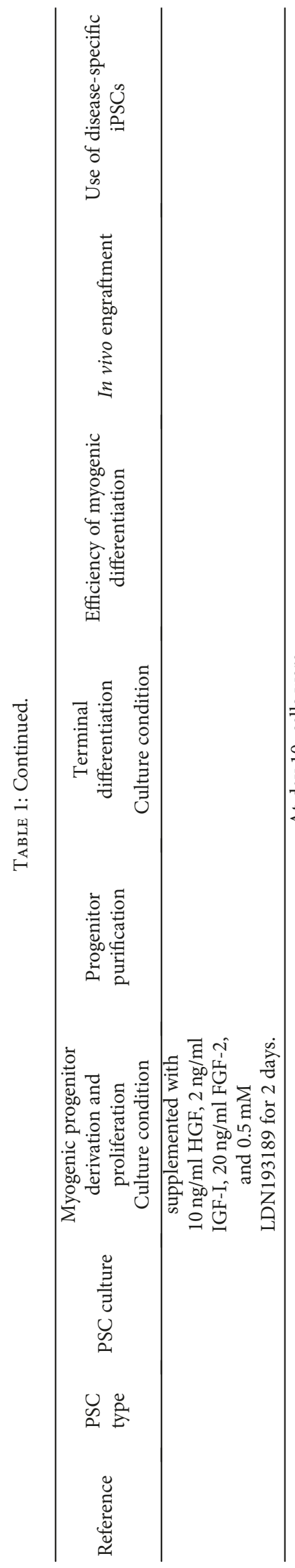

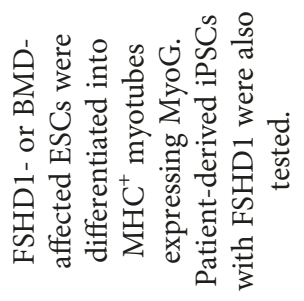

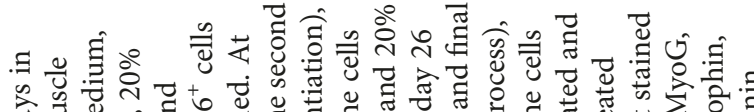

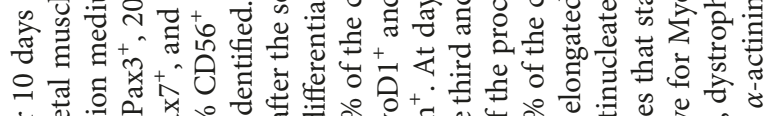

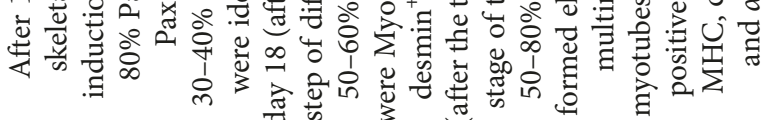

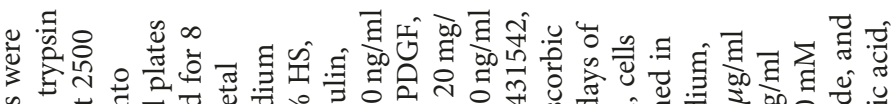

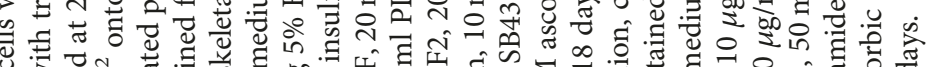

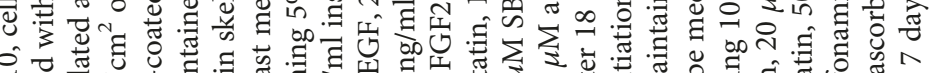

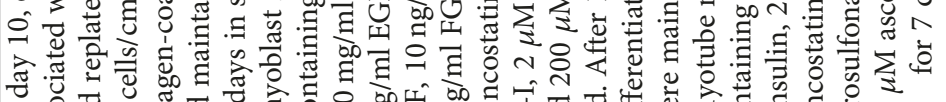

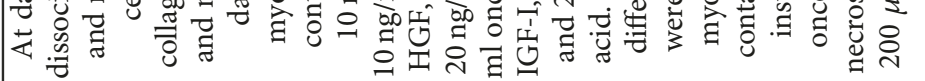

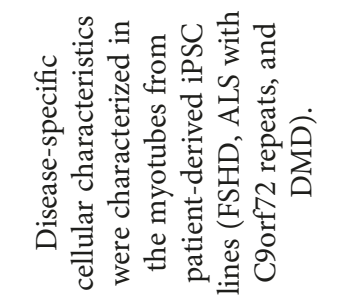

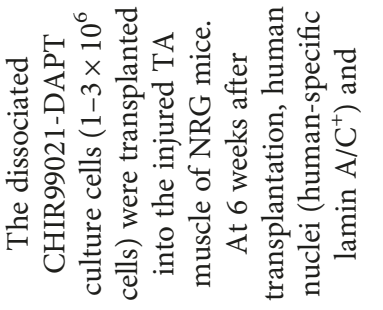

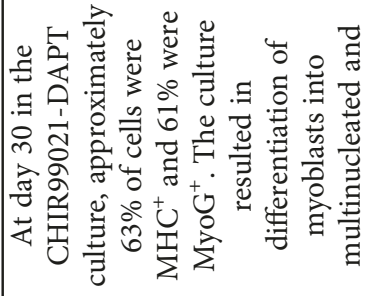

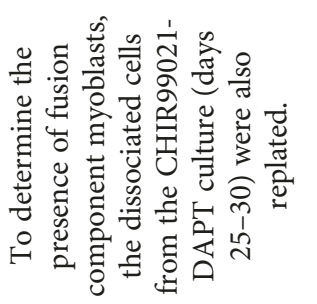

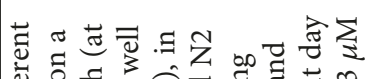

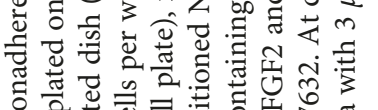

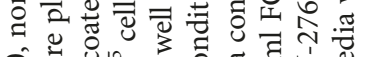

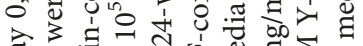

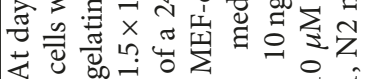

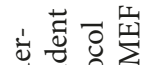

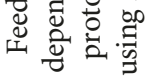

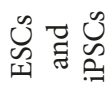

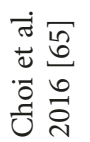




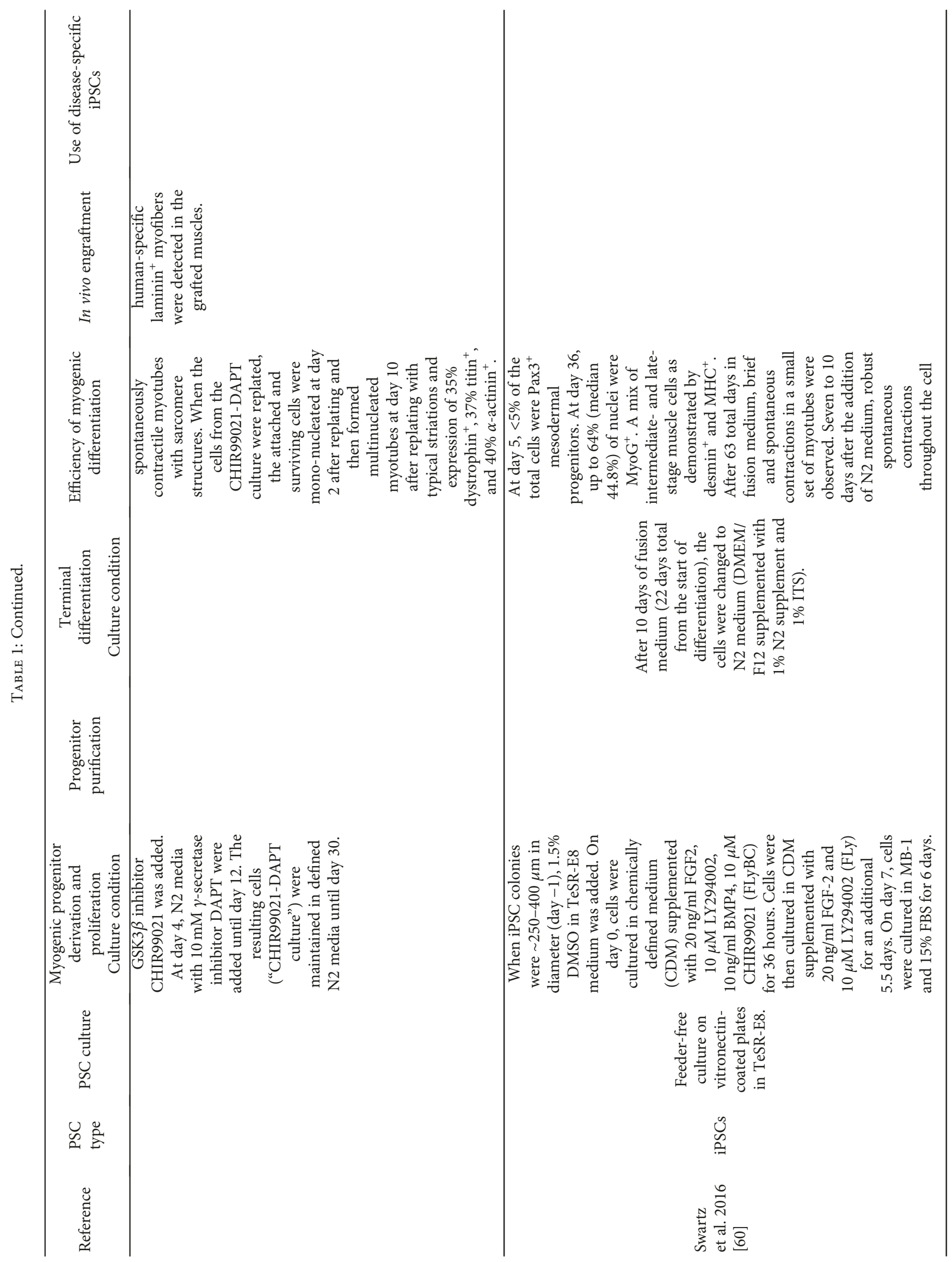




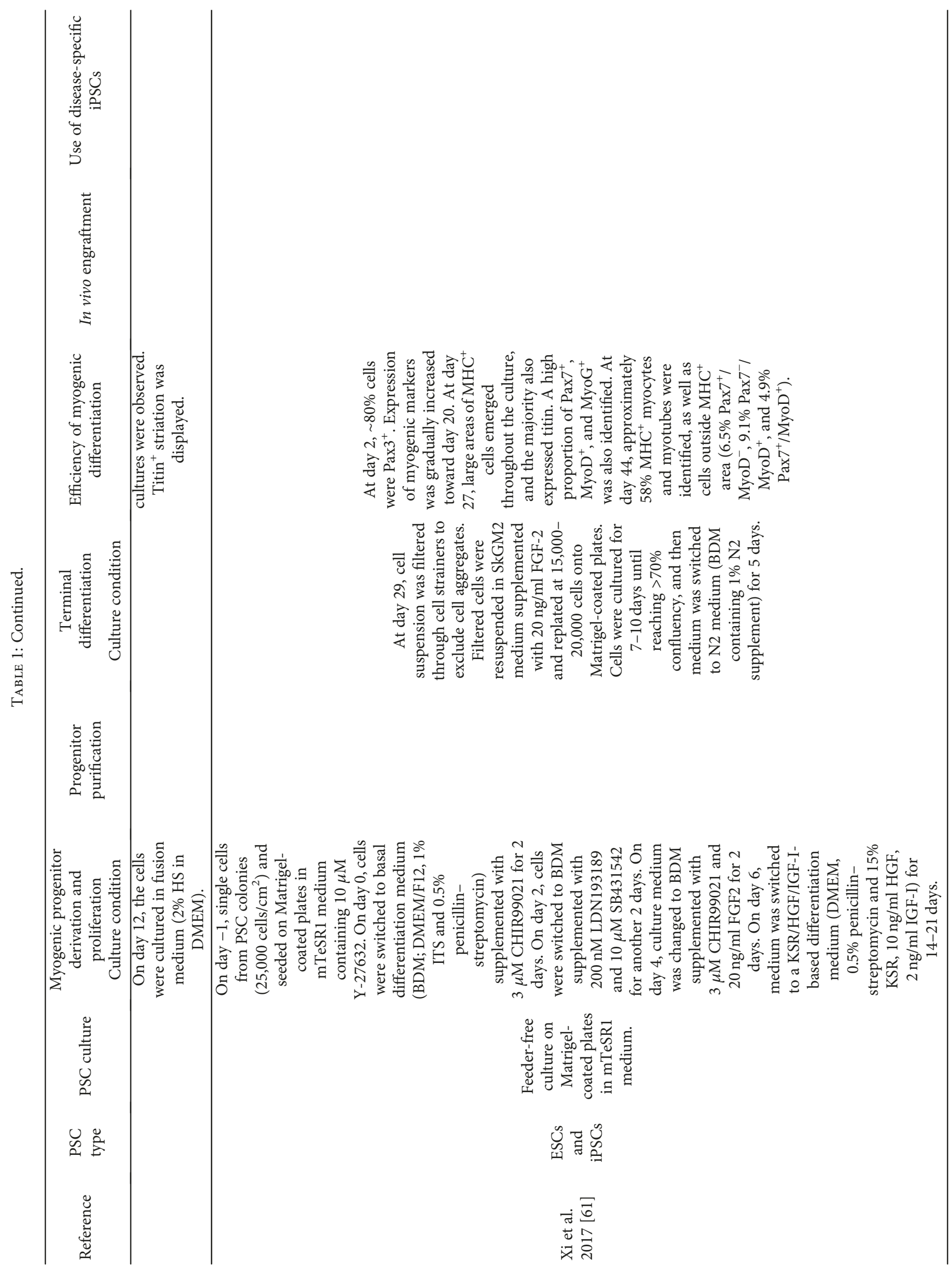




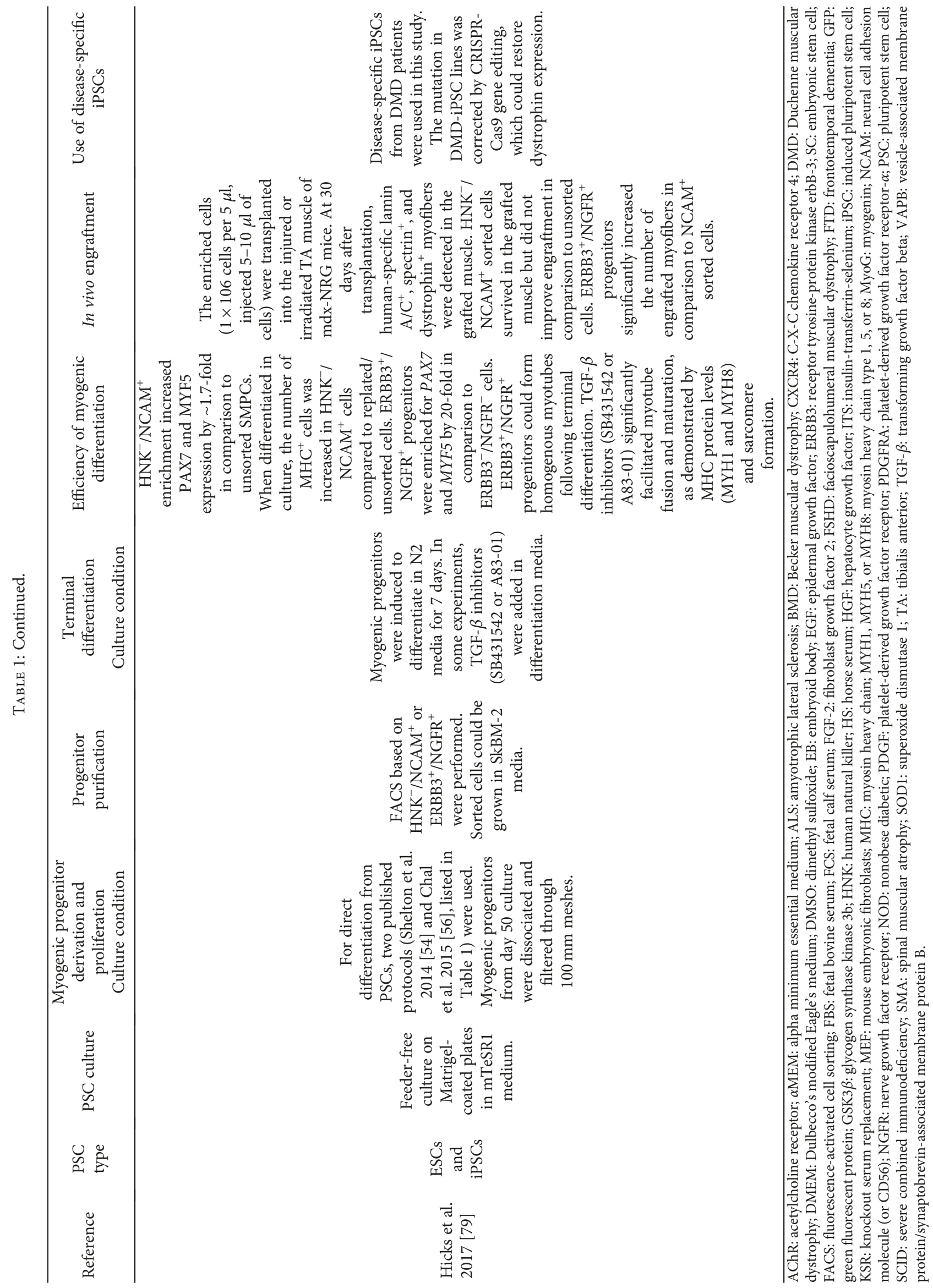




\section{Challenges for the Derivation of Skeletal Myocytes from Human PSCs Using Transgene-Free Methods}

The evaluation of differentiation efficiency and myocyte maturity has been inconsistent between studies that focus on differentiating skeletal myocytes from stem cells. It would be of great benefit to the field to establish standards for these evaluations in order to more directly compare differentiation methods. Another challenge facing the field is that in vitro stem cell-derived skeletal myocytes often have an embryonic or perinatal phenotype. Additional bioengineering methods may be necessary in order to achieve skeletal muscle that is fully mature and therefore more physiologically relevant to in vivo skeletal muscle. In this section, we will discuss existing concerns of the current methods for preparing skeletal myocytes and myogenic progenitors from human PSCs, specifically related to transgene-free methods. However, several concerns are also applicable to transgene methods.

4.1. Differentiation Efficiency. Compared to when using transgene protocols, differentiation efficiency of skeletal myocytes overall still remains low when using transgenefree approaches. In order for the field to move forward toward goals of disease modeling, drug testing, and therapeutic development, differentiation efficiency should be improved. Currently, there is a wide range of reported efficiencies due to differences in reporting methods and the definitions used to describe the maturity of myogenic cell types. It is common to use stains for myogenic markers such as Pax7, MyoD, myogenin, and MHC. However, there is variation in how these stains are used to determine efficiency. Some protocols claim a very high efficiency rate of myogenic differentiation but often use a pooled percentage of Pax7, MyoD, myogenin, and/or MHC-positive cells. Others with lower efficiency may only be using one of the markers, which could be different from the marker chosen in another study. Along with the usage of immunocytochemistry for MHC, the counting of $\mathrm{MHC}^{+}$cells in a field of view, the number of nuclei per myocyte, and the percentage of nuclei within myocytes (fusion index) have all been used to evaluate differentiation efficiency. Often, myocyte density and/or differentiation efficiency varies across a culture. Therefore, it is important to report the number of fields counted and how they were selected-specifically noting how bias was controlled. Overall, there is a need to standardize methods of calculating differentiation efficiency in order to facilitate comparisons between differentiation protocols.

4.2. Defining and Measuring the Extent of Myotube Maturation. In recent years, there have been a number of culture methods developed that yield MHC-positive skeletal myocytes from human pluripotent cells. Many of them require an extended culture period in comparison to methods for deriving other cell types. A method yielding myogenic progenitors or mature myocytes after a relatively short time would be of high significance to the field. However, it is also important to evaluate the maturity of cells yielded from rapid preparations. To date, it is difficult to compare the maturation state of myocytes generated by different methods due to differences in how each study defines maturity. Some focus on anatomical features, while others examine physiological functionality. Ideally, both aspects should be considered when evaluating myotube maturity. Studies taking an anatomical approach tend to use immunocytochemistry or electron microscopy to evaluate sarcomere formation and myofibril alignment as indicators of myotube maturity. Immunocytochemistry using antibodies against MHC or titin is a relatively accessible method to detect striations (Figure 2(b)); however, electron microscopy makes it possible to visualize sarcomeres at an ultrastructural level and examine sarcomeric organization and alignment (Figure 2(c)). It should be noted that in some preparations of maturing human PSC-derived myocytes, the results of immunocytochemical labeling of sarcomeric proteins (such as titin) may not correlate with ultrastructural results obtained through electron microscopy [67].

Some, but not all, studies take a physiological approach to determine myocyte maturation by examining the functionality of the cells. One method is to measure the frequency and coordination of spontaneous contractions observed in the differentiating myocytes. Contractions can be stimulated by a calcium flux or with addition of acetylcholine to the culture. Spontaneous contractions can also be observed shortly after culture medium changes $[59,67]$. To properly examine spontaneous contractions, it should be taken into consideration when the cells were last given fresh media or were supplemented with the compounds that can promote contractions. Electrophysiology has also been used to monitor contractions and record contraction frequency and strength in cultured myotubes $[48,68]$. Further, calcium imaging using dyes such as Fluo-3AM can be applied as an alternative method to electrophysiology. With a wide variety of methods currently being used, it is necessary to establish a preferred method of assessing physiological maturity of in vitro myocytes to better compare derivation methods. Another aspect of myocyte maturity is the fiber type expressed. During myogenesis, embryonic and slow type I MHC are expressed first. Then at later stages of maturation, myofibers develop glycolytic fast twitch MHC types IIa, IIb, and IIx [24, 69]. Commonly, MHC expression is examined using an antibody that reacts to all isoforms of MHC (such as MF20 clone), but a more detailed evaluation of MHC type would be useful for describing derived myocyte maturation.

In order to use human PSC-derived myocytes for in vitro modeling for adult-onset neuromuscular diseases, it is necessary to generate fully matured myotubes. However, iPSCderived skeletal myocytes prepared using current methods typically are of an embryonic or perinatal phenotype. In addition to better understanding signaling molecules and the timing required for generating mature myocytes, bioengineering techniques will be needed to create surfaces recognized by human PSC-derived myocytes as appropriate for growth and maturation. Differentiation efficiency can likely be improved by controlling features such as surface coatings, adhesion ligands, and/or growth surfaces that encourage directionality and elongation. For instance, micropatterned surfaces can give myocytes much needed directionality [70]. 
It is likely that most two-dimensional culture environments are not similar enough to in vivo and that three-dimensional constructs will become necessary to encourage further stages of maturation [67]. Cocultures with motor neurons may support myotube maturation, as stimulation is required for proper contractility in vivo [71]. In the absence of motor neurons, myotubes can be chemically stimulated to contract by adding acetylcholine to the culture [72]. Electrical stimulation can also induce contractions and enhance maturation of myotubes [49, 73]. Further, mechanical stimulation may accelerate muscle differentiation and maturation [74].

4.3. Cell Enrichment and Large-Scale Expansion. While the way we report the efficiency of myogenic differentiation is valuable, it is also important to improve upon current methods to gain a pure population of myogenic progenitors and skeletal myocytes in culture. When prepared by a transgene-free method, the cultures commonly contain a heterogeneous cell population with myocytes and other cell types. Such heterogeneity influences the efficiency of in vivo engraftment following transplantation [75]. In order to improve differentiation efficiency, there is a need for more precise definition of which signal molecules to use and the timing of their use, but improved cell sorting techniques will also be necessary to further enrich derived myocytes. Fluorophorelabeled progenitors can easily be purified by FACS, if genetic modification is used $[47,49]$. Also, several combinations of specific cell surface markers can be used to enrich myogenic progenitors and skeletal myocytes [55, 76-78]. Examples include combinations of CD $54^{+} /$integrin $\alpha 9 \beta 1^{+} / \mathrm{SDC}^{+}$[76], $\mathrm{CD}^{-} 5^{-} / \mathrm{CD} 11 \mathrm{~b}^{-} / \mathrm{GlyA}^{-} / \mathrm{CD} 31^{-} / \mathrm{CD} 34^{-} / \mathrm{CD} 56^{\text {int }} / \mathrm{ITGA}^{\mathrm{hi}}$ [77], $\mathrm{CD}^{+} 6^{+} / \mathrm{CD} 15^{-}$[78], $\mathrm{CXCR}^{+} / \mathrm{C}-\mathrm{MET}^{+}$[55], and $\mathrm{HNK}^{-} /$ NCAM $(\mathrm{CD} 56)^{+}[65,79]$. The most recent study indicated that a combination of two surface markers (ERBB3 and NGFR) can be applied to sufficiently purify a specific cellular population of human PSC-derived myogenic progenitors by FACS [79].

Another important consideration when developing derivation methods is whether they are adaptable to a large-scale expansion of myogenic progenitors and skeletal myocytes. Limited scalability seems to be a continued challenge among methods [75], which limits practical application and translation to patients as cell-based therapies. Often, cells are maintained in small quantities as a monolayer culture that is not always suitable for passaging. A recent study indicated that animal serum could promote cell expansion in PSC-derived myogenic progenitors, but the culture condition remained less defined [56]. However, a sphere-based culture may work to overcome this concern $[59,67]$. As demonstrated in our recent study, human PSC-derived spherical cultures can be expanded for several weeks with specific signaling molecule supplementation in the medium $[59,67]$.

\section{Conclusions}

Valuable knowledge regarding the differentiation of myogenic progenitors and myotubes from human PSCs has been gradually accumulating [1, 3, 80-82]. Signaling molecules significantly contribute to generating a sufficient number of myogenic progenitors and myocytes from human ESCs and iPSCs without genetic modification. In addition to directing and enhancing differentiation of myogenic cells using signaling molecules, recent bioengineering approaches such as two-dimensional or three-dimensional culture, micropatterning, controlled stiffness, and mechanical, chemical, or electrical stimulation have enabled us to more accurately mimic the physiological environment of cultured cells while improving throughput, accuracy, and efficiency of in vitro analyses. A combination of signaling molecules and bioengineering approaches may further enhance the differentiation and maturation of human PSCs-derived myotubes for use in disease modeling, drug testing, and therapeutic development. Finally, in vitro cell models should represent similar morphological and physiological characteristics compared to tissues in vivo. In the skeletal muscle, fully mature myotubes have well-organized sarcomeres and the ability to contract in response to stimulation. In order to assess the maturity of human PSC-derived myotubes, it will be necessary to evaluate them using both anatomical and functional approaches.

\section{Conflicts of Interest}

The authors declare that there is no conflict of interest regarding the publication of this paper.

\section{Acknowledgments}

The first author (Nunnapas Jiwlawat) would like to thank the Royal Thai Government Scholarship for the financial support. This work was supported by grants from the ALS Association (15-IIP-201, Masatoshi Suzuki), NIH/NINDS (R01NS091540, Masatoshi Suzuki), and the University of Wisconsin Foundation (Masatoshi Suzuki).

\section{References}

[1] T. Hosoyama, J. Van Dyke, and M. Suzuki, "Applications of skeletal muscle progenitor cells for neuromuscular diseases," American Journal of Stem Cells, vol. 1, no. 3, pp. 253-263, 2012.

[2] I. H. Park, N. Arora, H. Huo et al., "Disease-specific induced pluripotent stem cells,” Cell, vol. 134, no. 5, pp. 877-886, 2008.

[3] Y. Kodaka, G. Rabu, and A. Asakura, "Skeletal muscle cell induction from pluripotent stem cells," Stem Cells International, vol. 2017, Article ID 1376151, 16 pages, 2017.

[4] J. Chal and O. Pourquie, "Making muscle: skeletal myogenesis in vivo and in vitro," Development, vol. 144, no. 12, pp. 21042122, 2017.

[5] B. Jostes, C. Walther, and P. Gruss, "The murine paired box gene, $P a x 7$, is expressed specifically during the development of the nervous and muscular system," Mechanisms of Development, vol. 33, no. 1, pp. 27-37, 1990.

[6] M. Goulding, A. Lumsden, and A. J. Paquette, "Regulation of Pax-3 expression in the dermomyotome and its role in muscle development," Development, vol. 120, no. 4, pp. 957-971, 1994.

[7] M. Buckingham and F. Relaix, "PAX3 and PAX7 as upstream regulators of myogenesis," Seminars in Cell \& Developmental Biology, vol. 44, pp. 115-125, 2015. 
[8] D. Sassoon, G. Lyons, W. E. Wright et al., "Expression of two myogenic regulatory factors myogenin and MyoD1 during mouse embryogenesis," Nature, vol. 341, no. 6240, pp. 303307, 1989.

[9] Y. Cinnamon, N. Kahane, I. Bachelet, and C. Kalcheim, “The sub-lip domain-a distinct pathway for myotome precursors that demonstrate rostral-caudal migration," Development, vol. 128, no. 3, pp. 341-351, 2001.

[10] J. C. Kiefer and S. D. Hauschka, "Myf-5 is transiently expressed in nonmuscle mesoderm and exhibits dynamic regional changes within the presegmented mesoderm and somites I-IV," Developmental Biology, vol. 232, no. 1, pp. 77-90, 2001.

[11] M. Zhang and I. S. McLennan, "During secondary myotube formation, primary myotubes preferentially absorb new nuclei at their ends," Developmental Dynamics, vol. 204, no. 2, pp. 168-177, 1995.

[12] S. Tajbakhsh, U. Borello, E. Vivarelli et al., "Differential activation of Myf5 and MyoD by different Wnts in explants of mouse paraxial mesoderm and the later activation of myogenesis in the absence of Myf5," Development, vol. 125, no. 21, pp. 4155-4162, 1998.

[13] H. Amthor, B. Christ, and K. Patel, "A molecular mechanism enabling continuous embryonic muscle growth-a balance between proliferation and differentiation," Development, vol. 126, no. 5, pp. 1041-1053, 1999.

[14] B. A. Parr, M. J. Shea, G. Vassileva, and A. McMahon, "Mouse Wnt genes exhibit discrete domains of expression in the early embryonic CNS and limb buds," Development, vol. 119, no. 1, pp. 247-261, 1993.

[15] M. Ikeya and S. Takada, "Wnt signaling from the dorsal neural tube is required for the formation of the medial dermomyotome," Development, vol. 125, no. 24, pp. 4969-4976, 1998.

[16] R. van Amerongen and R. Nusse, "Towards an integrated view of Wnt signaling in development," Development, vol. 136, no. 19, pp. 3205-3214, 2009.

[17] A. E. Chen, D. D. Ginty, and C. M. Fan, "Protein kinase A signalling via CREB controls myogenesis induced by Wnt proteins," Nature, vol. 433, no. 7023, pp. 317-322, 2004.

[18] U. Borello, M. Coletta, S. Tajbakhsh et al., "Transplacental delivery of the Wnt antagonist Frzb1 inhibits development of caudal paraxial mesoderm and skeletal myogenesis in mouse embryos," Development, vol. 126, no. 19, pp. 42474255, 1999.

[19] A. Suzuki, R. C. Pelikan, and J. Iwata, "WNT/ $\beta$-catenin signaling regulates multiple steps of myogenesis by regulating step-specific targets," Molecular and Cellular Biology, vol. 35, no. 10, pp. 1763-1776, 2015.

[20] C. Xu, M. Tabebordbar, S. Iovino et al., “A zebrafish embryo culture system defines factors that promote vertebrate myogenesis across species," Cell, vol. 155, no. 4, pp. 909-921, 2013.

[21] C. Patsch, L. Challet-Meylan, E. C. Thoma et al., "Generation of vascular endothelial and smooth muscle cells from human pluripotent stem cells," Nature Cell Biology, vol. 17, no. 8, pp. 994-1003, 2015.

[22] M. Shelton, A. Kocharyan, J. Liu, I. S. Skerjanc, and W. L. Stanford, "Robust generation and expansion of skeletal muscle progenitors and myocytes from human pluripotent stem cells," Methods, vol. 101, pp. 73-84, 2016.

[23] R. L. Johnson, E. Laufer, R. D. Riddle, and C. Tabin, "Ectopic expression of sonic hedgehog alters dorsal-ventral patterning of somites," Cell, vol. 79, no. 7, pp. 1165-1173, 1994.
[24] X. Feng, E. G. Adiarte, and S. H. Devoto, "Hedgehog acts directly on the zebrafish dermomyotome to promote myogenic differentiation," Developmental Biology, vol. 300, no. 2, pp. 736-746, 2006.

[25] C. F. Bentzinger, Y. X. Wang, and M. A. Rudnicki, "Building muscle: molecular regulation of myogenesis," Cold Spring Harbor Perspectives in Biology, vol. 4, no. 2, 2012.

[26] C. Chiang, Y. Litingtung, E. Lee et al., "Cyclopia and defective axial patterning in mice lacking sonic hedgehog gene function," Nature, vol. 383, no. 6599, pp. 407-413, 1996.

[27] A. G. Borycki, L. Mendham, and C. P. Emerson Jr., "Control of somite patterning by sonic hedgehog and its downstream signal response genes," Development, vol. 125, no. 4, pp. $777-$ 790, 1998.

[28] K. A. Scata, D. W. Bernard, J. Fox, and J. L. Swain, "FGF receptor availability regulates skeletal myogenesis," Experimental Cell Research, vol. 250, no. 1, pp. 10-21, 1999.

[29] L. L. Tortorella, D. J. Milasincic, and P. F. Pilch, "Critical proliferation-independent window for basic fibroblast growth factor repression of myogenesis via the p42/p44 MAPK signaling pathway," The Journal of Biological Chemistry, vol. 276, no. 17, pp. 13709-13717, 2001.

[30] M. Lagha, J. D. Kormish, D. Rocancourt et al., "Pax3 regulation of FGF signaling affects the progression of embryonic progenitor cells into the myogenic program," Genes \& Development, vol. 22, no. 13, pp. 1828-1837, 2008.

[31] R. Stewart, L. Flechner, M. Montminy, and R. Berdeaux, "CREB is activated by muscle injury and promotes muscle regeneration," PLoS One, vol. 6, no. 9, article e24714, 2011.

[32] O. Pourquie, M. Coltey, C. Breant, and N. M. le Douarin, "Control of somite patterning by signals from the lateral plate," Proceedings of the National Academy of Sciences of the United States of America, vol. 92, no. 8, pp. 3219-3223, 1995.

[33] E. Hirsinger, D. Duprez, C. Jouve, P. Malapert, J. Cooke, and O. Pourquié, "Noggin acts downstream of Wnt and sonic hedgehog to antagonize BMP4 in avian somite patterning," Development, vol. 124, no. 22, pp. 4605-4614, 1997.

[34] J. Gerhart, J. Elder, C. Neely et al., "MyoD-positive epiblast cells regulate skeletal muscle differentiation in the embryo," The Journal of Cell Biology, vol. 175, no. 2, pp. 283-292, 2006.

[35] H. Wang, C. Zang, X. S. Liu, and J. C. Aster, "The role of Notch receptors in transcriptional regulation," Journal of Cellular Physiology, vol. 230, no. 5, pp. 982-988, 2015.

[36] A. C. Rios, O. Serralbo, D. Salgado, and C. Marcelle, "Neural crest regulates myogenesis through the transient activation of NOTCH," Nature, vol. 473, no. 7348, pp. 532-535, 2011.

[37] S. Jarriault, C. Brou, F. Logeat, E. H. Schroeter, R. Kopan, and A. Israel, "Signalling downstream of activated mammalian Notch," Nature, vol. 377, no. 6547, pp. 355-358, 1995.

[38] T. J. Brennan, D. G. Edmondson, L. Li, and E. N. Olson, "Transforming growth factor beta represses the actions of myogenin through a mechanism independent of DNA binding," Proceedings of the National Academy of Sciences of the United States of America, vol. 88, no. 9, pp. 3822-3826, 1991.

[39] C. Krueger and F. M. Hoffmann, "Identification of retinoic acid in a high content screen for agents that overcome the anti-myogenic effect of TGF-beta-1," PLoS One, vol. 5, no. 11, article e15511, 2010.

[40] H. Lee, C. Haller, C. Manneville et al., "Identification of small molecules which induce skeletal muscle differentiation in embryonic stem cells via activation of the Wnt and inhibition 
of Smad2/3 and sonic hedgehog pathways," Stem Cells, vol. 34, no. 2, pp. 299-310, 2016.

[41] D. Horbelt, J. H. Boergermann, A. Chaikuad et al., "Small molecules dorsomorphin and LDN-193189 inhibit myostatin/ GDF8 signaling and promote functional myoblast differentiation," The Journal of Biological Chemistry, vol. 290, no. 6, pp. 3390-3404, 2015.

[42] J. Zhu, Y. Li, A. Lu et al., "Follistatin improves skeletal muscle healing after injury and disease through an interaction with muscle regeneration, angiogenesis, and fibrosis," The American Journal of Pathology, vol. 179, no. 2, pp. 915930, 2011.

[43] C. Brun, O. Monestier, S. Legardinier, A. Maftah, and V. Blanquet, "Murine GASP-1 N-glycosylation is not essential for its activity on $\mathrm{C} 2 \mathrm{C} 12$ myogenic cells but alters Its secretion," Cellular Physiology and Biochemistry, vol. 30, no. 3, pp. 791-804, 2012.

[44] S. E. Tollefsen, R. Lajara, R. H. McCusker, D. R. Clemmons, and P. Rotwein, "Insulin-like growth factors (IGF) in muscle development. Expression of IGF-I, the IGF-I receptor, and an IGF binding protein during myoblast differentiation," The Journal of Biological Chemistry, vol. 264, no. 23, pp. 1381013817, 1989.

[45] H. H. Vandenburgh, P. Karlisch, J. Shansky, and R. Feldstein, "Insulin and IGF-I induce pronounced hypertrophy of skeletal myofibers in tissue culture," American Journal of PhysiologyCell Physiology, vol. 260, no. 3, pp. C475-C484, 1991.

[46] C. Serra, S. Bhasin, F. Tangherlini et al., "The role of GH and IGF-I in mediating anabolic effects of testosterone on androgen-responsive muscle," Endocrinology, vol. 152, no. 1, pp. 193-206, 2011.

[47] R. Darabi, R. W. Arpke, S. Irion et al., "Human ES- and iPSderived myogenic progenitors restore DYSTROPHIN and improve contractility upon transplantation in dystrophic mice," Cell Stem Cell, vol. 10, no. 5, pp. 610-619, 2012.

[48] G. Skoglund, J. Laine, R. Darabi, E. Fournier, R. Perlingeiro, and N. Tabti, "Physiological and ultrastructural features of human induced pluripotent and embryonic stem cell-derived skeletal myocytes in vitro," Proceedings of the National Academy of Sciences of the United States of America, vol. 111, no. 22, pp. 8275-8280, 2014.

[49] A. Tanaka, K. Woltjen, K. Miyake et al., "Efficient and reproducible myogenic differentiation from human iPS cells: prospects for modeling Miyoshi myopathy in vitro," PLoS One, vol. 8, no. 4, article e61540, 2013.

[50] T. Yasuno, K. Osafune, H. Sakurai et al., "Functional analysis of iPSC-derived myocytes from a patient with carnitine palmitoyltransferase II deficiency," Biochemical and Biophysical Research Communications, vol. 448, no. 2, pp. 175-181, 2014.

[51] R. Abujarour, M. Bennett, B. Valamehr et al., "Myogenic differentiation of muscular dystrophy-specific induced pluripotent stem cells for use in drug discovery," Stem Cells Translational Medicine, vol. 3, no. 2, pp. 149-160, 2014.

[52] S. M. Maffioletti, M. F. Gerli, M. Ragazzi et al., "Efficient derivation and inducible differentiation of expandable skeletal myogenic cells from human ES and patient-specific iPS cells," Nature Protocols, vol. 10, no. 7, pp. 941-958, 2015.

[53] T. Awaya, T. Kato, Y. Mizuno et al., "Selective development of myogenic mesenchymal cells from human embryonic and induced pluripotent stem cells," PLoS One, vol. 7, no. 12, article e51638, 2012.
[54] M. Shelton, J. Metz, J. Liu et al., "Derivation and expansion of PAX7-positive muscle progenitors from human and mouse embryonic stem cells," Stem Cell Reports, vol. 3, no. 3, pp. 516-529, 2014.

[55] B. Borchin, J. Chen, and T. Barberi, "Derivation and FACSmediated purification of PAX3+/PAX7+ skeletal muscle precursors from human pluripotent stem cells," Stem Cell Reports, vol. 1, no. 6, pp. 620-631, 2013.

[56] J. Chal, M. Oginuma, Z. Al Tanoury et al., "Differentiation of pluripotent stem cells to muscle fiber to model Duchenne muscular dystrophy," Nature Biotechnology, vol. 33, no. 9, pp. 962-969, 2015.

[57] L. Caron, D. Kher, K. L. Lee et al., "A human pluripotent stem cell model of facioscapulohumeral muscular dystrophyaffected skeletal muscles," Stem Cells Translational Medicine, vol. 5, no. 9, pp. 1145-1161, 2016.

[58] Y. Hwang, S. Suk, S. Lin et al., "Directed in vitro myogenesis of human embryonic stem cells and their in vivo engraftment," PLoS One, vol. 8, no. 8, article e72023, 2013.

[59] T. Hosoyama, J. V. McGivern, J. M. Van Dyke, A. D. Ebert, and M. Suzuki, "Derivation of myogenic progenitors directly from human pluripotent stem cells using a sphere-based culture," Stem Cells Translational Medicine, vol. 3, no. 5, pp. 564-574, 2014.

[60] E. W. Swartz, J. Baek, M. Pribadi et al., "A novel protocol for directed differentiation of C9orf72-associated human induced pluripotent stem cells into contractile skeletal Myotubes," Stem Cells Translational Medicine, vol. 5, no. 11, pp. 14611472, 2016.

[61] H. Xi, W. Fujiwara, K. Gonzalez et al., "In vivo human somitogenesis guides somite development from hPSCs," Cell Reports, vol. 18, no. 6, pp. 1573-1585, 2017.

[62] E. Latres, A. R. Amini, A. A. Amini et al., "Insulin-like growth factor-1 (IGF-1) inversely regulates atrophy-induced genes via the phosphatidylinositol 3-kinase/Akt/mammalian target of rapamycin (PI3K/Akt/mTOR) pathway," The Journal of Biological Chemistry, vol. 280, no. 4, pp. 2737-2744, 2005.

[63] J. Tureckova, E. M. Wilson, J. L. Cappalonga, and P. Rotwein, "Insulin-like growth factor-mediated muscle differentiation: collaboration between phosphatidylinositol 3-kinase-Akt-signaling pathways and myogenin," The Journal of Biological Chemistry, vol. 276, no. 42, pp. 39264-39270, 2001.

[64] J. Chal, Z. Al Tanoury, M. Hestin et al., "Generation of human muscle fibers and satellite-like cells from human pluripotent stem cells in vitro," Nature Protocols, vol. 11, no. 10, pp. 1833-1850, 2016.

[65] I. Y. Choi, H. Lim, K. Estrellas et al., "Concordant but varied phenotypes among Duchenne muscular dystrophy patientspecific myoblasts derived using a human iPSC-based model," Cell Reports, vol. 15, no. 10, pp. 2301-2312, 2016.

[66] T. Barberi, M. Bradbury, Z. Dincer, G. Panagiotakos, N. D. Socci, and L. Studer, "Derivation of engraftable skeletal myoblasts from human embryonic stem cells," Nature Medicine, vol. 13, no. 5, pp. 642-648, 2007.

[67] S. Jiwlawat, E. Lynch, J. Glaser et al., "Differentiation and sarcomere formation in skeletal myocytes directly prepared from human induced pluripotent stem cells using a sphere-based culture," Differentiation, vol. 96, pp. 70-81, 2017.

[68] X. Guo, K. Greene, N. Akanda et al., "In vitro differentiation of functional human skeletal myotubes in a defined system," Biomaterials Science, vol. 2, no. 1, pp. 131-138, 2014. 
[69] J. Talbot and L. Maves, "Skeletal muscle fiber type: using insights from muscle developmental biology to dissect targets for susceptibility and resistance to muscle disease," Wiley Interdisciplinary Reviews: Developmental Biology, vol. 5, no. 4, pp. 518-534, 2016.

[70] M. R. Salick, B. N. Napiwocki, J. Sha et al., "Micropattern width dependent sarcomere development in human ESCderived cardiomyocytes," Biomaterials, vol. 35, no. 15, pp. 4454-4464, 2014.

[71] S. Ostrovidov, V. Hosseini, S. Ahadian et al., "Skeletal muscle tissue engineering: methods to form skeletal myotubes and their applications," Tissue Engineering. Part B, Reviews, vol. 20, no. 5, pp. 403-436, 2014.

[72] L. Rao, Y. Qian, A. Khodabukus, T. Ribar, and N. Bursac, "Engineering human pluripotent stem cells into a functional skeletal muscle tissue," Nature Communications, vol. 9, no. 1, p. 126, 2018

[73] S. Ahadian, S. Ostrovidov, V. Hosseini et al., "Electrical stimulation as a biomimicry tool for regulating muscle cell behavior," Organogenesis, vol. 9, no. 2, pp. 87-92, 2013.

[74] S. Rangarajan, L. Madden, and N. Bursac, "Use of flow, electri$\mathrm{cal}$, and mechanical stimulation to promote engineering of striated muscles," Annals of Biomedical Engineering, vol. 42, no. 7, pp. 1391-1405, 2014.

[75] J. Kim, A. Magli, S. S. K. Chan et al., "Expansion and purification are critical for the therapeutic application of pluripotent stem cell-derived myogenic progenitors," Stem Cell Reports, vol. 9, no. 1, pp. 12-22, 2017.

[76] A. Magli, T. Incitti, J. Kiley et al., "PAX7 targets, CD54, integrin $\alpha 9 \beta 1$, and SDC2, allow isolation of human ESC/iPSCderived myogenic progenitors," Cell Reports, vol. 19, no. 13, pp. 2867-2877, 2017.

[77] A. Castiglioni, S. Hettmer, M. D. Lynes et al., "Isolation of progenitors that exhibit myogenic/osteogenic bipotency in vitro by fluorescence-activated cell sorting from human fetal muscle," Stem Cell Reports, vol. 2, no. 1, pp. 92-106, 2014.

[78] D. F. Pisani, N. Clement, A. Loubat et al., "Hierarchization of myogenic and adipogenic progenitors within human skeletal muscle," Stem Cells, vol. 28, no. 12, pp. 2182-2194, 2010.

[79] M. R. Hicks, J. Hiserodt, K. Paras et al., "ERBB3 and NGFR mark a distinct skeletal muscle progenitor cell in human development and hPSCs," Nature Cell Biology, vol. 20, no. 1, pp. 46$57,2018$.

[80] H. M. Blau, "Cell therapies for muscular dystrophy," The New England Journal of Medicine, vol. 359, no. 13, pp. 1403-1405, 2008.

[81] F. Rinaldi and R. C. Perlingeiro, "Stem cells for skeletal muscle regeneration: therapeutic potential and roadblocks," Translational Research, vol. 163, no. 4, pp. 409-417, 2014.

[82] I. Roca, J. Requena, M. J. Edel, and A. B. Alvarez-Palomo, "Myogenic precursors from iPS cells for skeletal muscle cell replacement therapy," Journal of Clinical Medicine, vol. 4, no. 12 , pp. 243-259, 2015. 


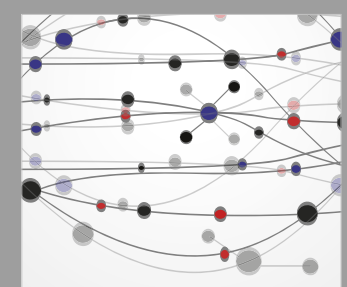

The Scientific World Journal
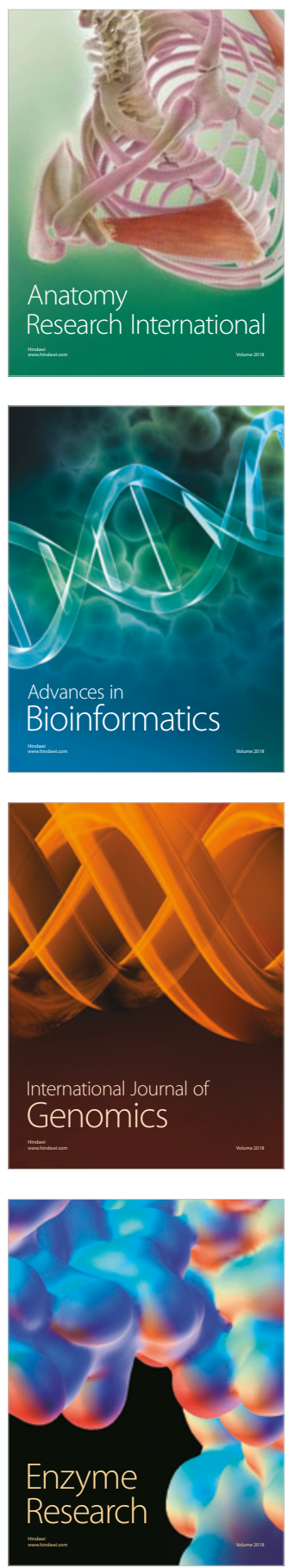
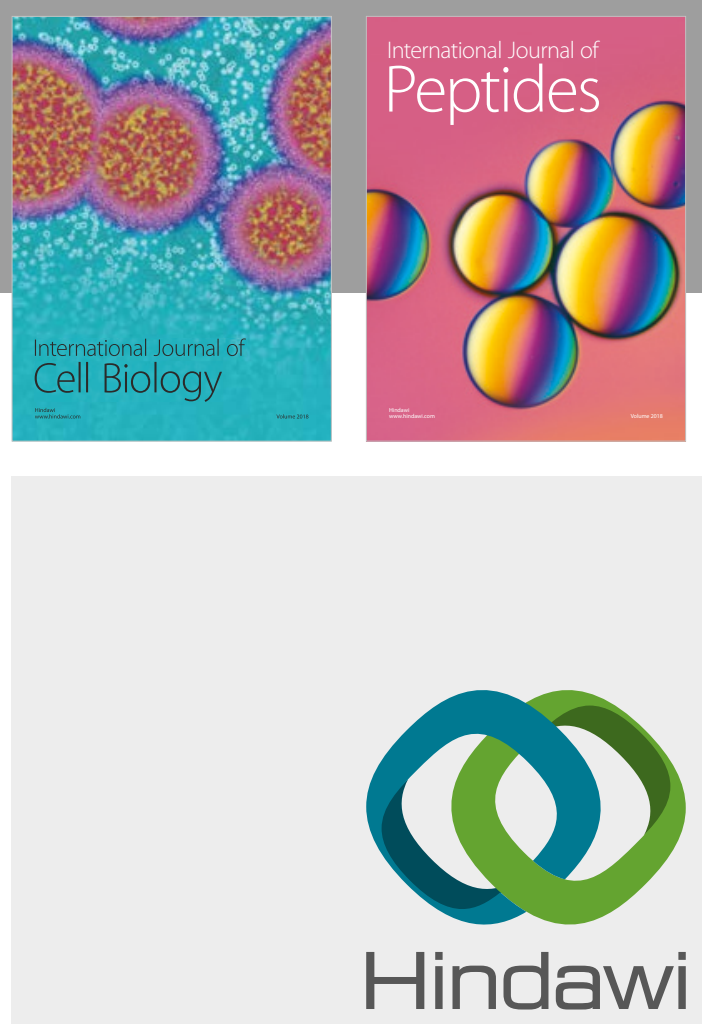

Submit your manuscripts at

www.hindawi.com
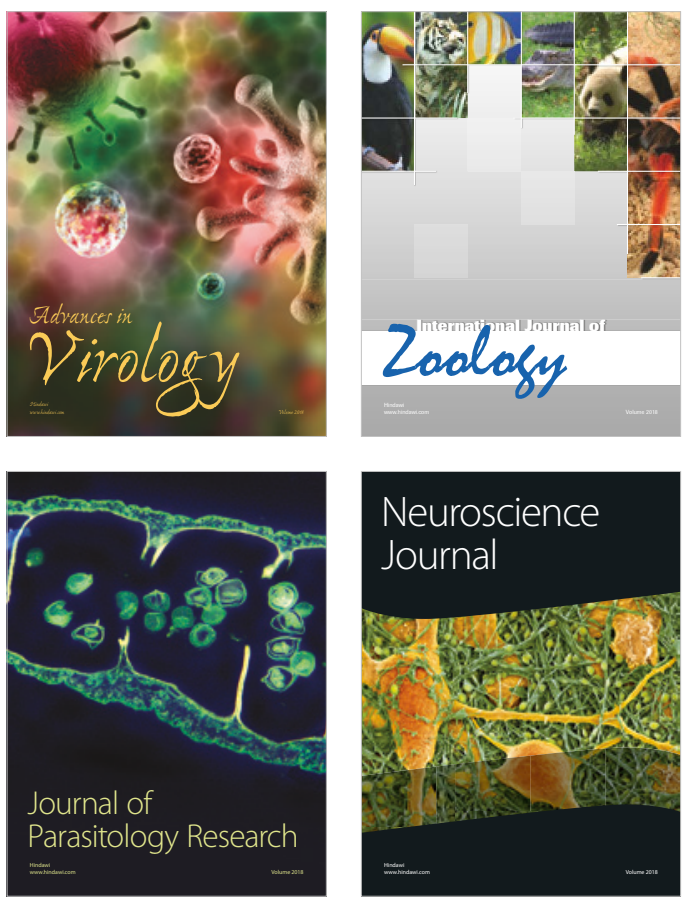
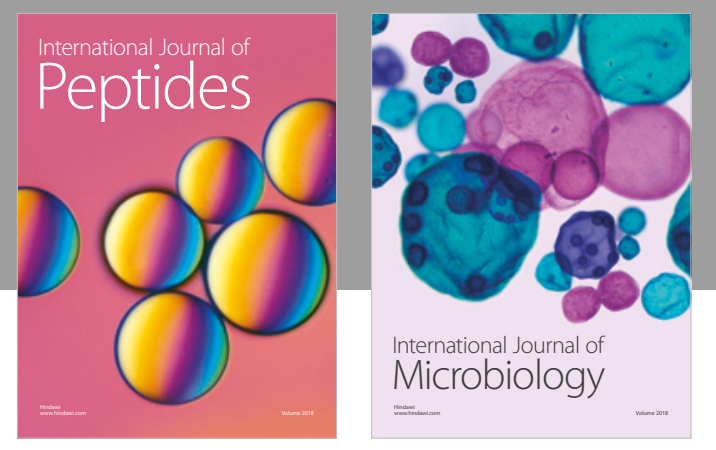

nternational Journal of Microbiology
Journal of
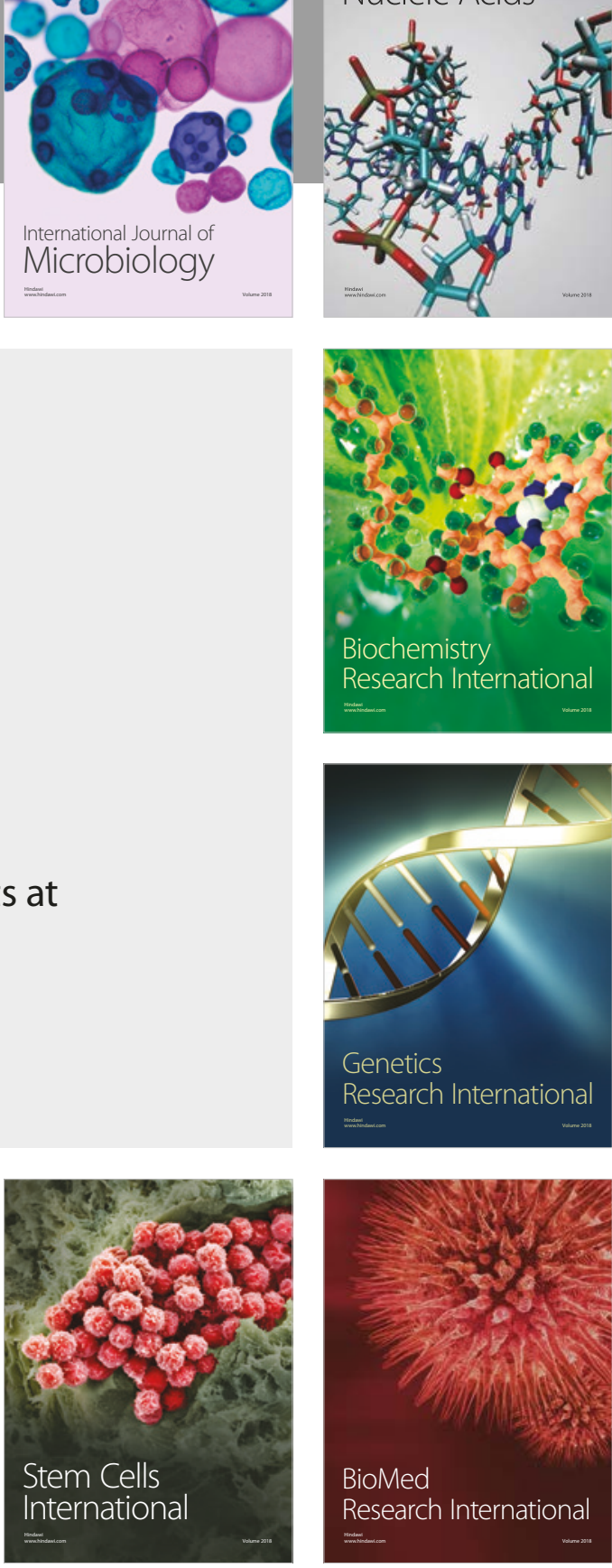
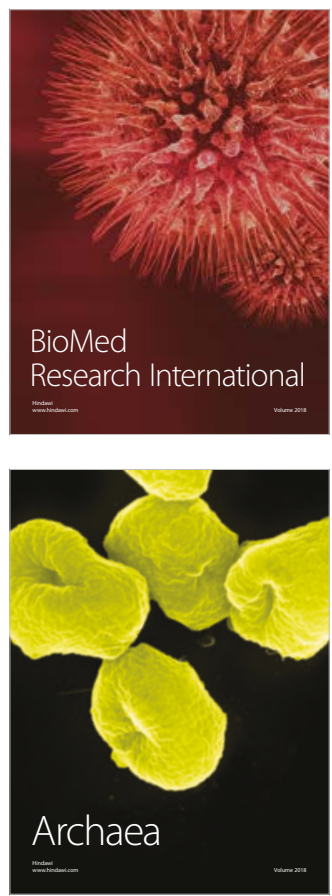